\title{
A HUGE BROAD LIGAMENT FIBROID WITH DEGENERATIVE CHANGES: A RARE CASE REPORT
}

\author{
Neetu Purwar1, Shobha Dwivedi², Brijendra Nath Tripathi ${ }^{3}$ \\ ${ }^{1}$ Lecturer, Department of Pathology, Ganesh Shankar Vidyarthi Memorial Medical College, Kanpur. \\ ${ }^{2}$ Associate Professor, Department of Pathology, Ganesh Shankar Vidyarthi Memorial Medical College, Kanpur. \\ ${ }^{3}$ Ex-Professor, Department of Pathology, Ganesh Shankar Vidyarthi Memorial Medical College, Kanpur.
}

\begin{abstract}
Broad ligament is a very uncommon site for presentation of leiomyoma with a very low incidence rate. Because of its overall low incidence rate, it poses both clinical and radiological difficulty in differentiating with an ovarian tumour. We are presenting a rare case of leiomyoma of broad ligament in a 48-year-old female patient who presented with complaints of lower abdominal pain of long duration associated with menstruation along with bladder complaints. On clinical and radiological examination, it was found to be a left-sided mass in the pelvic region suspected to be a tubo-ovarian mass. On laparotomy, there was a huge mass measuring $18 \times 14 \times 12$ $\mathrm{cm}$ in the left-sided broad ligament attached to the lateral wall of uterus. On histopathology, it was confirmed to be a soft tissue tumour - leiomyoma. We present this case because of its rarity and the diagnostic difficulties posed.
\end{abstract}

\section{KEYWORDS}

Broad Ligament, Leiomyoma, Laparotomy

HOW TO CITE THIS ARTICLE: Purwar N, Dwivedi S, Tripathi BN. A huge broad ligament fibroid with degenerative changes: A rare case report. J. Evolution Med. Dent. Sci. 2016;5(36):2171-2172, DOI: 10.14260/jemds/2016/506.

\section{INTRODUCTION}

Leiomyomas are most often benign tumours of the female genital tract. Uterine leiomyomas are the most common myomas accounting for approximately $20-30 \%$ of cases in females less than 35 years. Cervical fibroids account for $3 \%$ to $8 \%$ of uterine leiomyoma. Leiomyoma of the broad ligament is extremely rare.[1] It is the most common solid tumour of the broad ligament, but incidence of broad ligament fibroid is less than $1 \%{ }^{[2]}$ On account of its rarity, it poses specific diagnostic difficulties and may be responsible for erroneous diagnosis and management.[3] Here, we present a rare case of huge broad ligament fibroid mimicking ovarian tumour creating diagnostic difficulties.

\section{CASE REPORT}

We are reporting a case of a 48-year-old female, admitted to our hospital with complaints of abdominal mass along with pain in abdomen during menstruation since 1 year. She had two full-term normal vaginal deliveries with no history of any abortion. Her menstrual history was 5-6 days/30 days, regular, associated with pain in lower abdomen since 1 year. On presentation, the pain was of severe intensity. There was no significant past history. On abdominal palpation, a large soft to firm, slight tender mass was felt in the left lower pelvic region. On per speculum examination, the cervix and vagina were healthy. Her haematological parameters were within normal limits. Urine examination revealed few pus cells and few epithelial cells. Urine culture was negative. The liver and renal function tests were within normal limits. On Ultrasonography, a large solid hypoechoic heterogenous lesion extending from pelvis (left adnexa) up to the left hypochondriac region was noted.

Financial or Other, Competing Interest: None.

Submission 20-03-2016, Peer Review 16-04-2016,

Acceptance 21-04-2016, Published 05-05-2016.

Corresponding Author:

Neetu Purwar,

D-15,

Ganesh Shankar Vidyarthi Memorial Medical College,

Kanpur-208002.

E-mail: neetu.purwar@gmail.com

DOI: $10.14260 /$ jemds/2016/506
No obvious internal foci of calcification were seen. Left ovary could not be seen separately from the lesion. Radiologically, suspicious of left adnexal mass-ovarian tumour was given. Exploratory laparotomy with removal of left side broad ligament mass along with adherent left fallopian tube and left ovary was done and sent for histopathology.

\section{Gross Findings}

On gross examination, adherent left fallopian tube and left ovary were unremarkable. Left side broad ligament shows a single large circumscribed mass measuring $18 \times 14 \times 12 \mathrm{~cm}$ and weighing $2.5 \mathrm{~kg}$ (Fig.1). External surface showed congested blood vessels and nodularity. On cut section, mass was grey white, firm with whorled appearance. A small thick stalk with blood vessel was seen attached to superolateral part of the broad ligament.

\section{Microscopic Findings}

Multiple sections from the broad ligament mass showed a benign soft tissue tumour composed of spindle cells arranged in interlacing bundles, sheets and whorling pattern. Individual cells were elongated with cigar shaped nuclei and moderate amount of eosinophilic cytoplasm (Fig 2). The nuclei were uniform. Mitotic activity was sparse. Nuclear palisading, hyaline change and myxoid changes were noted focally. On the above mentioned findings, a histopathological diagnosis of benign soft tissue tumour-leiomyoma of the broad ligament with secondary changes was given. Sections through the left ovary and fallopian tube were unremarkable.

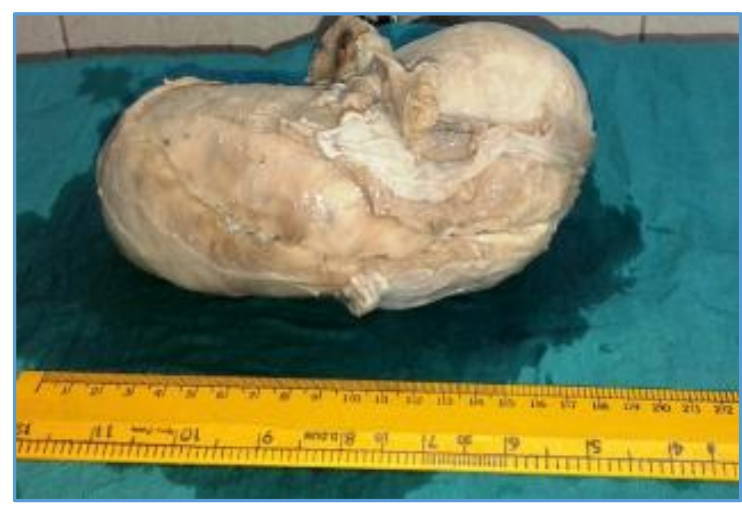

Fig. 1: Microphotograph Showing a Large Mass Arising From Broad Ligament with Attached Normal Ovary and Fallopian Tube 


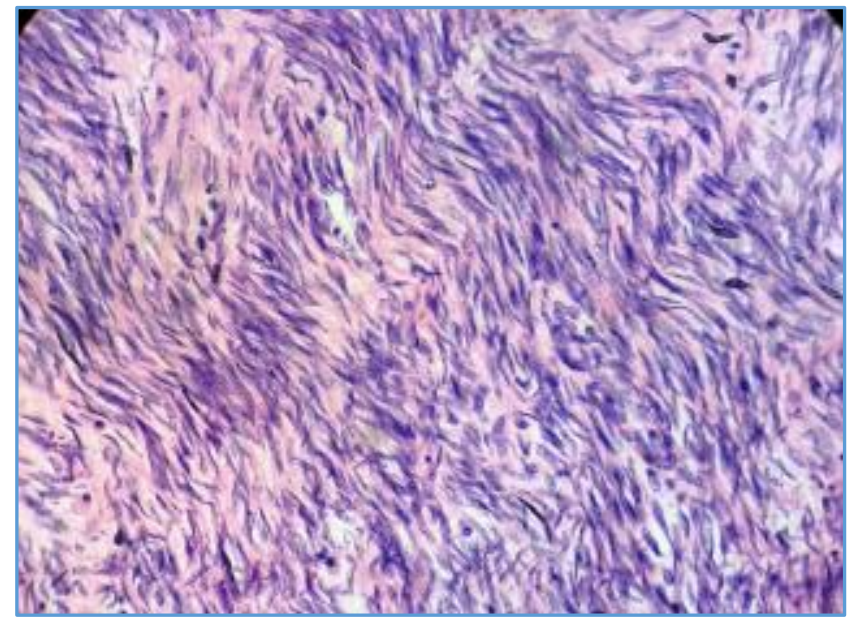

Fig. 2: Photomicrograph Showing Elongated, Cigar Shaped Nuclei with Moderate Amount of Eosinophilic Cytoplasm. (H \& E STAIN, 400X)

\section{DISCUSSION}

The most common tumour of female pelvis is the leiomyoma with a prevalence of $20 \%-30 \%$. They rarely occur at extrauterine location. Leiomyoma of uterus arises from smooth muscles of uterus. The size, number and location determine their clinical presentation. It can be single or multiple, with size variation from small to huge. They can be intramural, submucosal, or subserosal which may be pedunculated and simulate adnexal mass. In $50 \%$ of cases, they are asymptomatic. ${ }^{[4]}$ The extra-uterine location of leiomyoma can be in broad ligament, ovary, urinary bladder, urethra, vulva, vagina or anywhere there is smooth muscle.

Other variants in growth patterns can be diffuse peritoneal leiomyomatosis, intravenous leiomyomatosis, parasitic leiomyoma or benign metastasising leiomyomatosis, where they can be found even in lung due to haematogenous dissemination and retroperitoneal leiomyomas.[5] Among extra-uterine fibroids, broad ligament fibroids generally achieve enormous size and generally present with pressure symptom like bladder and bowel dysfunction.[6] We report a case of true broad ligament fibroid measured about $18 \times 14 \times 12$ $\mathrm{cm}$. This case is reported for its rarity and the diagnostic difficulties in differentiating ovarian tumour and giant fibroid.

These benign tumours are usually asymptomatic. However, if the leiomyoma reaches significant size, it can push uterus to contralateral side or it can potentially compress the surrounding pelvis structure and manifest clinically with various sign and symptoms.[7] They give rise to greater surgical difficulty by virtue of their relative inaccessibility and close proximity to the bladder and uterus. Enlargement causes upward displacement of the uterus and the fibroid may become impacted in the pelvis, causing urinary retention and ureteric obstruction.[6] Most common secondary changes in fibroids are degenerations, infections, haemorrhage, necrosis and sarcomatous changes.

Myxoid degeneration is a rare complication of benign fibroid, where presence of cystic changes mimics the metastatic malignant ovarian tumour.[7]
Ultrasonographic features play an important role in diagnosis of parasitic and pedunculated leiomyoma. Since parasitic leiomyoma are separated from the uterus, they are easily mistaken for adnexal tumours such as ovarian tumours.[8] The differential diagnosis for broad ligament leiomyoma includes masses from ovarian origin-benign or malignant, broad ligament cyst, lymphadenopathy and tuboovarian masses. In our case on clinical and radiological investigation, it was suspicious of ovarian neoplasm.

The serum levels of cancer antigen CA-125 were done which is in normal range. Elevated cancer marker CA-125 levels may point to metastatic ovarian malignancies. But CA125 is also raised in endometriosis, endometriomas, serous benign tumours and cystic teratomas which make difficult to diagnose preoperatively. Histopathology plays an important role in diagnosis for such cases. [9] Primary leiomyosarcoma in broad ligament is rarely reported.[7] So, proper histopathological evaluation is important for patient management.

\section{CONCLUSION}

Broad ligament leiomyomas mimic ovarian tumours on clinical and radiological examination and it should be kept important differential diagnosis for such solid adnexal or ovarian mass. The diagnosis of broad ligament leiomyoma is difficult on clinical and radiological features owing to its rarity and unusual presentation. It is thus very important for the histopathologist to diagnose it unequivocally.

\section{REFERENCES}

1. Bhalla N. Tumours of the corpus uteri. In: Jeffcoate's principles of gynaecology. London: Arnold printers. $2001 ; 6^{\text {th }}$ ed:p 470.

2. Bakari F, Sulayman HU, Avidime S, et al. Huge broad ligament leiomyoma: a case report. Case Reports in Clinical Medicine and Healthcare 2015;4(2):55-9.

3. Yuel VI, Kaur V. Broad ligament fibroid: an unusual presentation. J K science 2006;8(4):217-8.

4. Malik R, Agarwal G. Large cystic degenerating broad ligament leiomyoma masquerading as ovarian malignancy!. JCR 2015;5:486-9.

5. Fasih N, Prasad Shanbhogue AK, Macdonald DB, et al. Leiomyomas beyond the uterus: unusual location, rare manifestations. RSNA Radiographics 2008;28(7):1931-48.

6. Goel N, Laddad M. Rare case of giant broad ligament fibroid with cervical fibroid mimicking ovarian tumour: interesting case report. International Journal of Recent Trends in Science And Technology ISSN 2277-2812 E-ISSN 2249-8109, 2014;10(2):208-9.

7. Jagtap Sunil V, Gupta A, Kshirsagar NS. Broad ligament leiomyoma mimicking as ovarian tumour. Sch J App Med Sci 2014;2(1C):258-60.

8. Yeh HC, Kaplan M, Deligdisch L. Parasitic and pedunculated leiomyomas: ultrasonographic features. J Ultrasound Med 1999;18(11):789-94.

9. Jacobs I, Bast RC. The CA 125 tumour associated antigen: a review of the literature. Hum Reprod 1989;4(1):1-12. 